\title{
Construction des centres d'urgence - une aide à la planification
}

Robert Sieber

\section{Pour l'instant, il n'existe en Suisse aucune directive pertinente ni norme de construction concernant les questions de planification et de technique de construction relatives à la réalisation d'un CU}

Il n'existe pas de conflits d'intérêts.

Correspondance: Dr Robert Sieber Capo Servizio ProntoSoccorso Ospedale Regionale di Lugano Via Tesserete 46 CH-6903 Lugano robert.sieber@eoc.ch

\begin{abstract}
Introduction
Pour beaucoup d'hôpitaux, l'exploitation d'un centre d'urgence (CU) est une tâche essentielle. De nos jours, une partie considérable des patients hospitalisés le sont par le biais du CU. Quant à l'activité de médecine d'urgence ambulatoire, elle suit ces dernières années une courbe de croissance continue et aucun changement de cette tendance n'est visible à l'horizon. Les soins de base de médecine d'urgence à la population dans les zones urbaines sont de plus en plus supportés par les CU. C'est pourquoi, ceux-ci deviennent une priorité en matière de politique de la santé. Sans compter que la perception de la prestation globale de l'hôpital est en grande partie subjective et dépend de la réputation du CU. Celui-ci est donc un investissement capital pour l'avenir de l'hôpital et son image.
\end{abstract}




\begin{tabular}{|c|c|c|}
\hline \multicolumn{3}{|c|}{$\begin{array}{l}\text { Phases de planification du CU } \\
\text { (adapté d'après le règlement SIA } 112 \text { «Modèle de prestations», Société suisse des ingénieurs } \\
\text { et des architectes, www.sia.ch). }\end{array}$} \\
\hline Phases & Etapes & Aspects - contenus \\
\hline \multirow[t]{2}{*}{$\begin{array}{l}\text { Planification } \\
\text { stratégique }\end{array}$} & $\begin{array}{l}\text { Etude des besoins } \\
\text { en soins d'urgence }\end{array}$ & $\begin{array}{l}\text { - Conditions-cadres / besoins } \\
\text { - Concepts de soins existants } \\
\text { - Identification des partenaires }\end{array}$ \\
\hline & $\begin{array}{l}\text { Formulation des besoins } \\
\text { Solutions possibles }\end{array}$ & $\begin{array}{l}\text { - Objectifs / stratégies } \\
\text { - Liens avec le réseau de soins } \\
\text { - Synergies }\end{array}$ \\
\hline \multirow[t]{2}{*}{ Etudes préliminaires } & $\begin{array}{l}\text { Etudes de faisabilité } \\
\text { Idées de projet }\end{array}$ & $\begin{array}{l}\text { - Définition de la manière de } \\
\text { procéder et de I'organisation } \\
\text { des groupes de travail } \\
\text { - Elaboration des bases du projet }\end{array}$ \\
\hline & $\begin{array}{l}\text { Procédure de sélection } \\
\text { Critères d'évaluation }\end{array}$ & $\begin{array}{l}\text { - Définition du projet et choix } \\
\text { de mandataires selon profil } \\
\text { des exigences }\end{array}$ \\
\hline \multirow[t]{3}{*}{ Etude de projet } & $\begin{array}{l}\text { Avant-projet } \\
\text { (sélection de variantes) }\end{array}$ & $\begin{array}{l}\text { - Optimisation de la conception } \\
\text { et de l'économicité / efficience } \\
\text { - Esquisses/répartition des espaces }\end{array}$ \\
\hline & Projet de l'ouvrage & $\begin{array}{l}\text { - Optimisation du projet / coûts } \\
\text { - Définition des délais du projet } \\
\text { - Demande de crédit de construction }\end{array}$ \\
\hline & Autorisations & $\begin{array}{l}\text { - Demandes d'autorisation } \\
\text { - Contrôle des coûts / des délais } \\
\text { - Autorisation et crédit } \\
\text { de construction }\end{array}$ \\
\hline \multirow[t]{3}{*}{ Appel d'offres } & Appel d'offres & $\begin{array}{l}\text { - Procédure d'appel d'offres et d'octroi } \\
\text { (conditions-cadres légales) } \\
\text { - Planning (évent. délais de recours) }\end{array}$ \\
\hline & Evaluation des offres & $\begin{array}{l}\text { - Optimisation de l'économicité } \\
\text { (coupes et réductions) } \\
\text { - Modifications }\end{array}$ \\
\hline & Projet d'exécution & $\begin{array}{l}\text { - Elaboration des plans d'exécution } \\
\text { - Mise au point détaillée du projet } \\
\text { d'exécution } \\
\text { - Contrôle des coûts }\end{array}$ \\
\hline \multirow[t]{2}{*}{ Réalisation } & Exécution & $\begin{array}{l}\text { - Contrôle de l'exécution (cahier } \\
\text { des charges des constructeurs) } \\
\text { - Inspections par le groupe de base }\end{array}$ \\
\hline & $\begin{array}{l}\text { Mise en service } \\
\text { Achèvement du projet }\end{array}$ & $\begin{array}{l}\text { - Délais / transfert de ressources } \\
\text { - Optimisation } \\
\text { - Correction de défauts de construction }\end{array}$ \\
\hline
\end{tabular}

Les données brutes de la liste des espaces décrits en détail ont été discutées avec différents pairs, complétées et au besoin adaptées à nos particularités nationales. Une procédure de consultation a eu lieu auprès des participants du «Forum de médecine d'urgence hospitalière SSMUS»* et au sein du comité de la Communauté d'intérêts Soins d'urgence Suisse (SUS). Finalement, le document a été relu par un ingénieur en bâtiment et des spécialistes en gestion et maintenance de bâtiments.

Le «Forum de médecin d'urgence hospitalière SSMUS» existe depuis 2002 et il réunit les responsables des centres d'urgence de toutes les régions de la Suisse. Il se conçoit comme un forum de discussion et un organe d'experts pour les questions relatives à la médecine d'urgence hospitalière.

\section{Objectifs}

Les réflexions architectoniques présentées dans le document «Construction des centres d'urgence - une aide à la planification» sont destinées aux responsables des CU et leur servent à structurer leurs processus de planification (construction ou transformation) et à dé- finir l'indispensable «fil rouge» pour la planification. Les informations générales de planification mises à disposition permettent aux utilisateurs et aux constructeurs du CU de devenir des partenaires compétents et complémentaires.

Le guide place sciemment la partie des «utilisateurs» (personnel soignant et médecins) et les «opérateurs» (nettoyage, technique, logistique, etc.) au premier plan. Il s'agit des collaborateurs hospitaliers, qui connaissent bien les différentes tâches et compétences au sein d'un $C U$, mais sont moins versés dans les questions de construction.

Le document sert de norme pour le programme de répartition des espaces d'un centre d'urgence moderne et constituera également, à l'avenir, un argument important dans les discussions en matière de politique de la santé. Dans ce sens, il est le complément indispensable du document de base de K. Meier «Centres d'urgence hospitaliers: Recommandation structurelles et organisationnelles en matière d'assurance qualité», paru en 2005 dans l'édition $\mathrm{N}^{\circ} 34$ du Bulletin des médecins suisses.

\section{Limites}

Malgré la discussion avec des pairs de CU suisses, le document comprend de nombreux aspects issus de la littérature internationale. Des aspects qui ne correspondent pas toujours intégralement à notre réalité nationale et qui, par conséquent, sont forcément adaptés.

Vu l'étendue limitée du travail en question, les thèmes suivants n'ont été abordés que partiellement, voire pas du tout:

- les aspects techniques des éléments d'infrastructure (analyse des structures, installations, sécurité de fonctionnement électrique et électronique, dispositions de protection des installations radiologiques, etc.);

- l'équipement détaillé en outillage médical des CU;

- l'influence du choix du projet sur les coûts d'exploitation et les besoins en personnel;

- l'aspect des éventuels cabinets médicaux attenants au centre d'urgence.

Cela dit, ces aspects partiels ne sont pas d'importance mineure et sont traités dans d'autres documents.

C'est aux constructeurs qu'appartiennent la tâche et la compétence d'introduire dans le projet les détails de technique de construction qui n'ont pas été inclus, en les accompagnant des explications et informations nécessaires.

\section{Partie principale}

\section{Bases de planification et groupe de planification} Au début du projet, l'étude des besoins et l'analyse de la demande sont au premier plan. Même pour un CU, les besoins changent en permanence par rapport à l'activité menée auparavant, non seulement en raison 
de l'évolution épidémiologique mais aussi à cause des conditions politiques, de la structure de soins locale et du changement de comportement des patients. Les objectifs stratégiques ainsi obtenus sont harmonisés avec les objectifs d'amélioration de l'efficience et de l'efficacité des processus de fonctionnement des futurs CU.

Sous l'angle de vue technique des constructeurs (planificateur, responsable de la construction et ouvriers) du CU, les utilisateurs (comme souvent aussi l'administration de l'hôpital) sont dans la position des profanes. Autrement dit, le personnel du CU a peu de connaissances techniques spécifiques à la construction. Pourtant, les utilisateurs d'un CU ont une très grande expérience des exigences thérapeutiques et médico-techniques posées envers la fonctionnalité et l'infrastructure d'un CU.

Ainsi, lors d'une transformation ou d'une construction, deux catégories professionnelles sont concernées, qui ne parlent pas le même langage et souvent n'ont pas les mêmes objectifs. Les constructeurs s'intéressent en premier lieu à la technique et aux questions de coûts, alors que les utilisateurs visent une prise en charge efficiente des patients dans un lieu de travail fonctionnel.

La connaissance et la prise en compte des règles générales du travail en projet font partie intégrante d'une planification et réalisation réussies d'une construction. L'importance du groupe de travail «construction d'un nouveau CU» et du travail qu'il accomplit est souvent sous-estimée. L'efficience du travail du groupe de projet dépend de la direction de ce groupe et du fait de prendre en compte, directement ou non, tous ceux qui sont en relation avec l'activité du $\mathrm{CU}$, d'une manière étroite ou non (tab. 3). L'optimisation de la phase du projet tout comme son fonctionnement futur au quotidien ne réussissent cependant que si l'on inclut de manière conséquente - côté hôpital - les utilisateurs médicaux, médico-techniques et techniques du CU. Seul quelqu'un qui connaît les questions concernant les patients, le personnel et le matériel par son travail de tous les jours peut, grâce à ce «savoir d'initié», juger les propositions de planification quant à leur fonctionnalité.

\section{Indications architectoniques et techniques générales}

Cette partie contient une liste par mots-clés d'indications spécifiques de planification et d'ordre technique, dont il faut tenir compte à une ou plusieurs occasions dans la discussion relative à la planification et à l'exécution. Elle englobe un savoir de base architectonique qui a été adapté aux besoins des «profanes de la construction» du côté du CU. A cela, s'ajoutent des

Tableau 2

Détails du programme de répartition des espaces des centres d'urgence (extrait)

\begin{tabular}{|c|c|c|c|c|}
\hline $\mathrm{N}^{\circ}$ & Désignation / zone & Fonction de I'espace & Surface minimale & Remarques \\
\hline 5 & $\begin{array}{l}\text { Réanimation / Salle de } \\
\text { trauma / déchoquage }\end{array}$ & $\begin{array}{l}\text { Diagnostic et traitement } \\
\text { de malades graves / } \\
\text { de blessés }\end{array}$ & $\begin{array}{l}1 \text { espace de } 35 \mathrm{~m}^{2} \text {, si plusieurs } \\
\text { espaces: } 25 \mathrm{~m}^{2} / \text { espace } \\
\text { (1 espace / } 10000 \text { patients) }\end{array}$ & $\begin{array}{l}\text { - places de travail pour au moins } 5 \text { personnes } \\
\text { - espace de travail libre tout autour au moins } 1 \mathrm{~m} \text { (+ } 1 \mathrm{~m} \text { zone } \\
\text { latérale pour la circulation du personnel/des appareils) } \\
\text { - raccordements au gaz et électriques sur la paroi ou au plafond } \\
\text { - installation radiologique (fixe / mobile)/CT à proximité } \\
\text { immédiate (ou intégré) } \\
\text { - évent. analyseur des gaz du sang } \\
\text { - armoires murales et endroits fixes pour tous les appareils } \\
\text { - station PACS (ou négatoscope) } \\
\text { - réfrigérateur et armoire chauffante } \\
\text { - pharmacie } \\
\text { - équipement complet d'anesthésie }\end{array}$ \\
\hline $6 a$ & $\begin{array}{l}\text { Lit de traitement / } \\
\text { box (standard) }\end{array}$ & $\begin{array}{l}\text { Diagnostic et traitement } \\
\text { de patients atteints de } \\
\text { maladies ne mettant pas } \\
\text { leur vie en danger }\end{array}$ & $\begin{array}{l}\text { (10 à) } 12 \mathrm{~m}^{2} \\
\text { (env. } 3 \times 4 \mathrm{~m} \text { recommandé) }\end{array}$ & $\begin{array}{l}\text { - espace de traitement à une place } \\
\text { - fenêtre pouvant être fermée } \\
\text { - raccordements standards sur la paroi ou au plafond } \\
\text { (gaz, électricité) } \\
\text { - raccordement pour l'eau } \\
\text { - téléphone } \\
\text { - ordinateur (WLAN) } \\
\text { - moniteur relié au moniteur central (WLAN) } \\
\text { - lampe d'examen ( } 30000 \text { Lux) } \\
\text { - des deux côtés et au bas du lit / de la couchette, au moins } \\
1 \text { m de libre, longueur de l'espace au moins } 3 \text { m, surface } \\
\text { de base variable évent. en fonction de l'infrastructure } \\
\text { complémentaire de l'endroit }\end{array}$ \\
\hline $6 b$ & Salle de traitement & $\begin{array}{l}\text { Diagnostic et traitement } \\
\text { de patients atteints } \\
\text { de maladies ne mettant } \\
\text { pas leur vie en danger }\end{array}$ & 12 à $16 \mathrm{~m}^{2}$ & $\begin{array}{l}\text { - équipement de base comme (6a) } \\
\text { - espace de traitement à une place avec porte pouvant } \\
\text { être fermée } \\
\text { - fenêtre verrouillable pour cas un peu plus graves que (6a) } \\
\text { ou pour patients agités } \\
\text { - traitement de petites blessures également possible }\end{array}$ \\
\hline
\end{tabular}


Espace de soin «Pronto soccorso», Ospedale regionale di Lugano.

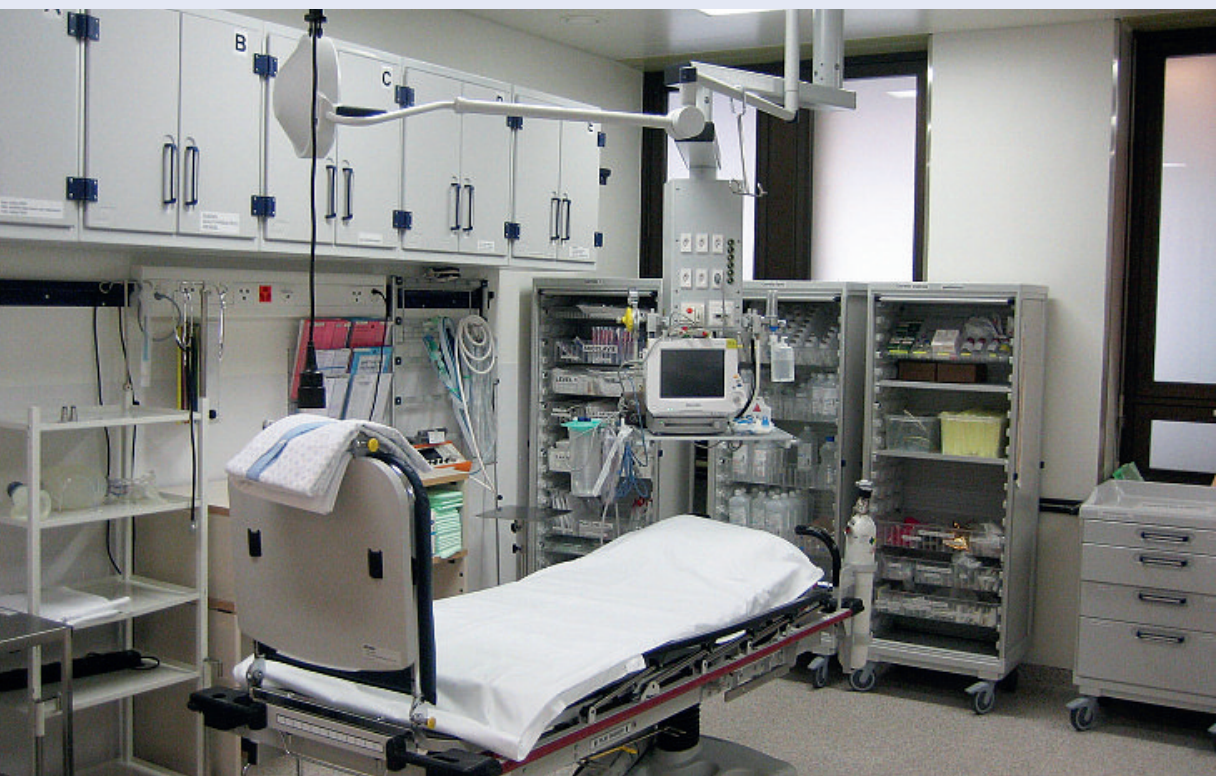

chiffres et des paramètres de référence. Ce sont là des informations de base indispensables pour le groupe de planification durant la phase d'étude de projet.

\section{Aspects architectoniques et techniques spéci-} fiques du programme de répartition des espaces Le programme de répartition des espaces reflète la liste des espaces du CU. Toutes les structures au sein du CU et les éventuelles parties fonctionnelles situées hors de celui-ci y sont énumérées. Ainsi, les aspects spécifiques les plus importants en termes de construction pour le programme de répartition des espaces d'un CU se trouvent résumées dans un tableau (voir extrait de celui-ci sous tab. 2).

Le projet ne dépend pas seulement de l'identification concrète des besoins, mais de sa qualité, qui se reflète aussi dans la transposition minutieuse des détails identifiés, effectuée par le groupe de travail sur les feuilles concernant les espaces effectifs.

\section{Perspectives}

La partie centrale du travail présenté, qui est orientée sur la pratique, ne doit pas être statique. Il s'agit plu-

\begin{tabular}{|c|c|}
\hline \multicolumn{2}{|c|}{ Médecin responsable CU (direction du groupe de base) } \\
\hline participants permanents & participants temporaires \\
\hline $\begin{array}{l}\text { - représentants médicaux des cliniques actifs } \\
\text { dans le CU } \\
\text { - personnel de soins du CU } \\
\text { - représentant de la direction } \\
\text { - chef de projet / architecte* }\end{array}$ & $\begin{array}{l}\text { - opérateurs (service technique, } \\
\text { nettoyage, transports) } \\
\text { - représentant de l'hygiène hospitalière } \\
\text { - représentant de l'assurance-qualité/ } \\
\text { gestion des risques } \\
\text { - experts externes/pairs }\end{array}$ \\
\hline
\end{tabular}

tôt de profiter d'autres expériences et développements au sens d'un «work in progress». Les futurs groupes de projet pourront communiquer leurs expériences et les rendre ainsi accessibles à un cercle plus large d'intéressés. En outre, toute remarque sera examinée et il en sera tenu compte dans une version révisée du document. Cet apport direct de la part des utilisateurs peut amener des améliorations ainsi que des compléments utiles.

Le document original «Construction des centres d'urgence - une aide à la planification» peut être téléchargé sur le site internet de la société (www.sgnor.ch). Une liste des références s'y trouve.

\section{Conclusions}

En Suisse également, il existe un besoin incontestable de créer des recommandations techniques et architectoniques pour la construction des centres d'urgence.

Les données et recommandations présentées ici correspondent à la pratique internationale.

Là où les conditions-cadres le permettent, le projet CU doit s'orienter sur les spécifications données. La transposition dans la pratique amènera un changement considérable des CU existants, car la plupart des structures actuelles ne correspondent pas à ces spécifications spatiales. L'adaptation des infrastructures impliquera forcément une augmentation des coûts. C'est pourquoi, les directions hospitalières et les groupes de projet n'échapperont pas au fait de devoir fixer des priorités et faire des coupes. Toutefois, certaines coupes pourraient à ce point diminuer la fonctionnalité et l'efficience qu'elles ne sont pas défendables. Notre objectif doit en effet être un projet de construction tourné vers le futur, mettant justement l'accent sur la fonctionnalité et l'efficience. Ce qui, à son tour, favorise la qualité des soins aux patients et la satisfaction des collaborateurs. L'amélioration simultanée de la productivité a, pour sa part, comme résultat, des gains économiques pour le CU.

Le chantier CU demeure un problème complexe. Mais, grâce aux instructions conviviales désormais disponibles et au savoir d'expert rassemblé, ce défi peut être surmonté plus facilement.

\section{Remarque et remerciements}

Des parties du document «Construction des centres d'urgence - une aide à la planification» ont été utilisées dans le cadre d'un travail de séminaire à la Haute école spécialisée de St-Gall (MAS in Health Service Management).

De précieux compléments et corrections ont été apportés au texte par la contribution technique d'Urs Wiederkehr-Kälin, dr ès sc. et ingénieur en bâtiment dipl. ETH/SIA (de GEWI Bauinformatik GmbH), auquel nous adressons ici nos remerciements.

Nous remercions également le Dr Kaspar Meier, en qualité d'initiateur du projet et les collègues du Forum de médecine d'urgence hospitalière SSMUS, pour la participation à la consultation. 